\title{
Thoracic NUT Carcinoma
}

National Cancer Institute

\section{Source}

National Cancer Institute. Thoracic NUT Carcinoma. NCI Thesaurus. Code C142781.

A highly aggressive, poorly differentiated carcinoma that arises from the thoracic structures. It is characterized by mutations and rearrang ement of the NUT gene. It usually presents at an advanced stage with pleuritic chest pain and pleural effusion, nonproductive cough, shortness of breath, and weigh loss. 